\title{
Importancia clínica de la Arteria umbilical única
} (A.U.U.).

\section{Informe preliminar}

\section{MIGUEL BUENO*}

HUMBERTO REY**

JAIRO DE LA CRUZ***

DAVID MCMURRAY****

- El cordón umbilical constituye la comunicación más importante entre el feto y la placenta. Normalmente está constituído por una vena que lleva sangre arterial al feto, y dos arterias que regresan a la placente con sangre venosa. Los tres vasos del cordón se encuentran suspendidos en la Gelatina de Wharton y rodeadas en conjunto por el amnios.

La ausencia de una arteria umbilical ha sido conocida desde la antiguedad. La primera referencia de esta anomalia congénita se encuentra en el ensayo "Una tantum artericum in fune Falopius vidit" escrito por Vesalio en 1530. (1) En 1620 Bauhin (2) describe otro caso, y 200 años después Baudelocque (3) en 1822 la describe en su libro "L'Arts des Accouchements". Poco

después, en 1830, Otto (4) publica la primera serie de 40 casos de A.U.U. En 1870 Hyrtl (5) describió la posible asociación de la A.U.U. con el sexo del niño y la severidad de otras malformaciones asociadas en el feto femenino.

Transcurrió casi otro siglo antes de que Benirschke y Brown (6) publicaran en 1955 los hallazgos realizados en 55 niños con A.U.U., de los cuales solo 13 sobrevivieron. Desde entonces han aparecido numerosas publicaciones sobre A.U.U. y su importancia como indicador de la posible coexistencia de otras malformaciones congénitas importantes. $(7,8,9,10,11,12)$.

De acuerdo con nuestra revisión de la literatura, solo se ha publicado un trabajo (13) sobre A.U.U. en América Latina, según puede observarse en el cuadro No. 1,

\begin{tabular}{|l|c|c|c|c|c|}
\hline AUTOR & AÑO & NUMERO DE CASOS & \% A.U.U & MALFORMACrON & MANGENITA \\
\hline Bernischke y Brown & 1955 & Est. Retrospectivo & 55 Caso & 49 \\
\hline Litle & 1958 & 1200 & 1.0 & 19 \\
\hline Bernischke & 1961 & 1500 & 1.0 & 47 \\
\hline Feingold et. al & 1964 & 6080 & 0.5 & - \\
\hline Papadatos y Paschos & 1965 & 7866 & 0.4 & 31 \\
\hline Masako, Seki y Strauss & 1966 & 3353 & 2.9 & 55 \\
\hline Segovia & 1967 & 10.000 & 0.6 & 13 \\
\hline Saigal y Srivastava & 1968 & 1000 & 0.6 & 50 \\
\hline Mullery Dchalleux & 1969 & 4600 & 0.6 & 7 \\
\hline Bruniquel & 1971 & 2100 & 0.7 & - \\
\hline Vlietink y Thieri & 1972 & 2572 & 1.1 & 39 \\
\hline Johnsonbaugh & 1973 & 1152 & 0.8 & 11 \\
\hline Froehlich y Fujikura & 1973 & 39.773 & 0.9 & 57 \\
\hline Nuestros resultados & 1975 & 3000 & 0.6 & 25 \\
\hline
\end{tabular}

* Profesor Titular, Departamento de Obstetricia y Ginecología, H.U.V.

* Profesor Auxiliar, Departamento de Pedia. tría, H.U.V.

*** Profesor Auxiliar, Departamento de Morfología, U. del V.

**** Investigador Asociado, ICMRT, Programa U del Valle - U de Tulane el cual muestra también otros trabajos al respecto, la incidencia de A.U.U. y la de malformaciones asociadas.

El propósito de nuestro trabajo es analizar la incidencia de la A.U.U. en nuestro medio y su posible asociación con otras 
malformaciones congénitas importantes.

El cuadro No. 2 muestra las malformaciones congénitas descritas en coexistencia con A.U.U.

$$
\begin{gathered}
\text { ANOMALIAS CONGENITAS } \\
\text { OBSER VADAS } \\
\text { EN } 33 \text { CASOS DE A. U. U. }
\end{gathered}
$$

Cuadro No. 2 (Según Seki y Strauss, 1964)

\begin{tabular}{|l|r|}
\hline SISTEMA CARDIOVASCULAR & 26 \\
\hline SISTEMA GENITOURINARIO & 16 \\
\hline SISTEMA GASTROINTESTINAL & 15 \\
\hline DEFECTOS NEUROLOGICOS & 9 \\
\hline SISTEMA MUSCULOESQUELETICO & 21 \\
\hline SISTEMA RESPIRATORIO & 14 \\
\hline $\begin{array}{c}\text { OTROS (Ano malías faciales, } \\
\text { auriculares u oculares) }\end{array}$ & 15 \\
\hline
\end{tabular}

\section{MATERIAL Y METODOS}

Se reviso en forma sistemática el cordón umbilical de todos los niños nacidos en el Servicio de Obstetricia del Hospital Universitario del Valle, en Cali, en el periodo comprendido entre el 1o. de Mayo y el 31 de Octubre de 1975. Ante la ausencia de una arteria umbilical, nos propusimos realizar los siguientes exámenes, según las anomalias mas frecuentemente descritas en la literatura.

1.- Examen físico cuidadoso, para buscar anomalias tales como tercera fontanela, malformaciones auriculares y oculares, labios leporino, paladar hendido, arteria esofogica, hernias, meningoceles y ano imperforado.

2.- Urografias excretora para buscar posibles anomalias urinarias.
3.-- Cariotipo y dermatoglifos para buscar anomalias cromosomicas tales como Trisomia y Mosaicismo.

4.- Inmunoglobulinas, como indicadora de una posible asociación con infección intrauterina, especialmente de tipo de la Enfermedad Citomegálica.

\section{HALLAZGOS}

Se encontraron 9 casos de A.U.U. en 3126 recien nacidos en nuestro hospital en el periodo previamente descrito.

El cuadro No. 3 muestra los antecedentes maternos en los 9 casos.

ANTECEDENTES MATERNOS EN

9 CAŚOS DE ARTERIA UMBILICIAL

UNICA Hospital Universitario, Cali

\begin{tabular}{|l|l|l|l|l|}
\hline ESO NO & EDAD & $(\mathrm{i} / \mathrm{P}$ & E.E. & PAT.ASOC \\
\hline 1 & & & & \\
\hline 2 & 19 & $4 / 4$ & 10 & R.P.M \\
\hline 3 & 23 & $1 / 1$ & 39 & R.P.M. \\
\hline 1 & 21 & $3 / 3$ & 39 & PREFCLANSIA SEVERA \\
\hline 5 & 26 & $1 / 4$ & 10 & NINGUNA \\
\hline 6 & 28 & $8 / 8$ & 10 & PRES. CARA \\
\hline 7 & 28 & $1 / 4$ & 39 & R.P.M. \\
\hline 8 & 33 & $5 / 5$ & 10 & NINGUNA \\
\hline 9 & 3.1 & $1 / 1$ & 38 & NINGUNA \\
\hline
\end{tabular}

EDAD. Como puede observarse 5 de las 9 madres estaban entre 20 y 30 años. La edad promedio fue de 26.2 años, y el rango entre 19 y 40 años.

GRAVIDEZ Y PARIDAD. Se observo que 8 de las 9 madres eran multigravidas con paridad de 4 o más. Solo una era primipara.

EDAD DEL EMBARAZO. Todos los embarazos eran de término.

EVENTOS FETALES EN 9 CASOS DE ARTERIA UMBILICAL UNICA Hospital Universitario Cali

Cuadro No. 4

\begin{tabular}{|c|c|c|c|c|l|l|l|l|c|}
\hline Caso No. & Sexo & Peso & Talla & Apgar & E. Fis. & Ur. Exc. & Ctipo & Oglifo. & Inmunog. \\
\hline 1 & $\wp$ & 3300 & 52 & 10 & Normal & N.R. & N.R. & N.R. & Elevadas \\
\hline 2 & $\delta$ & 3.460 & 51 & 8 & Normal & N,R. & N.R. & N.R. & Elevadas \\
\hline 3 & $q$ & 3.040 & 47 & 0 & Anencéfalo & N.R. & N.R. & N.R. & N.R. \\
\hline 4 & $q$ & 3.300 & 49 & 10 & Normal & N.R. & N.R. & N.R. & Normales \\
\hline 5 & $\delta$ & 3400 & 50 & 8 & Normal & N.R. & N.R. & N.R. & N.R. \\
\hline 6 & $\delta$ & 3200 & 50 & 10 & Normal & Normal & Normal & N.R. & Normal \\
\hline 7 & $\delta$ & 2640 & 47 & 10 & Normal & Normal & Normal & N.R. & N.R. \\
\hline 8 & $\delta$ & 3540 & 48 & 10 & Normal & N.R. & N.R. & N.R. & N.R. \\
\hline 9 & $\uparrow$ & 2950 & 51 & 10 & Normal & Agen.R.Izq. & N.R. & N.R. & N.R. \\
\hline
\end{tabular}


PATOLOGIA ASOCIADA. Cuatro de las nueve pacientes tuvieron ruptura prematura de membranas, dos tuvieron toxemia, y tres no presentaron patologia asociada al embarazo.

El cuadro No. 4 muestra los eventos fetales en los 9 casos de A.U.U. se puede observar lo siguiente:

SEXO. Cuatro niños eran del sexo femenino y cinco del sexo masculino.

PESO Y TALLA. Todos los niños tuvieron peso y talla correspondientes a fetos mææduros.

I. de APGAR. El Indice de Apgar fue de 10 en 6 niños. Dos niños tuvieron Apgar de 8 (uno con presentación de cara y otro con ruptura prematura de membranas). El feto anencefálico nacio muerto.

EXAMEN FISICO. Todos los niños fueron normales al examen físico, excepto el anencefálico.

UROGRAFIA EXCRETORA. Se realizo en 3 casos. En uno fue normal y en otro no se observó el riñón izquierdo. En el tercer caso no se observaron bien los ureteres, aunque los sistemas pielocaliciales aparecian normales.

CARIOTIPO. Se realizó en 2 casos, y en ambos fue normal.

DERMATOGLIFOS. La interpretación de los dermatoglifos en recien nacidos ofrece dificultades especiales, por lo cual se decidio aplazar su determinación.

INMUNOGLOBULINAS. Se realizaron en cuatro de los nueve casos. En dos casos los niveles de IGM estaban elevados y en los otros eran normales.

\section{DISCUSION}

El hallazgo de nueve casos de A.U.U. en los 3126 niños analizados por nosotros, arroja una incidencia de $0.3 \%$, menor que en la mayoria de los trabajos publicados. Nuestros resultados, aun incompletos, muestran la coexistencia de dos casos de malformaciones congénitas importantes: un caso de anencefaléa y otro de agenesia renal izquierda, lo cual esta de acuerdo con
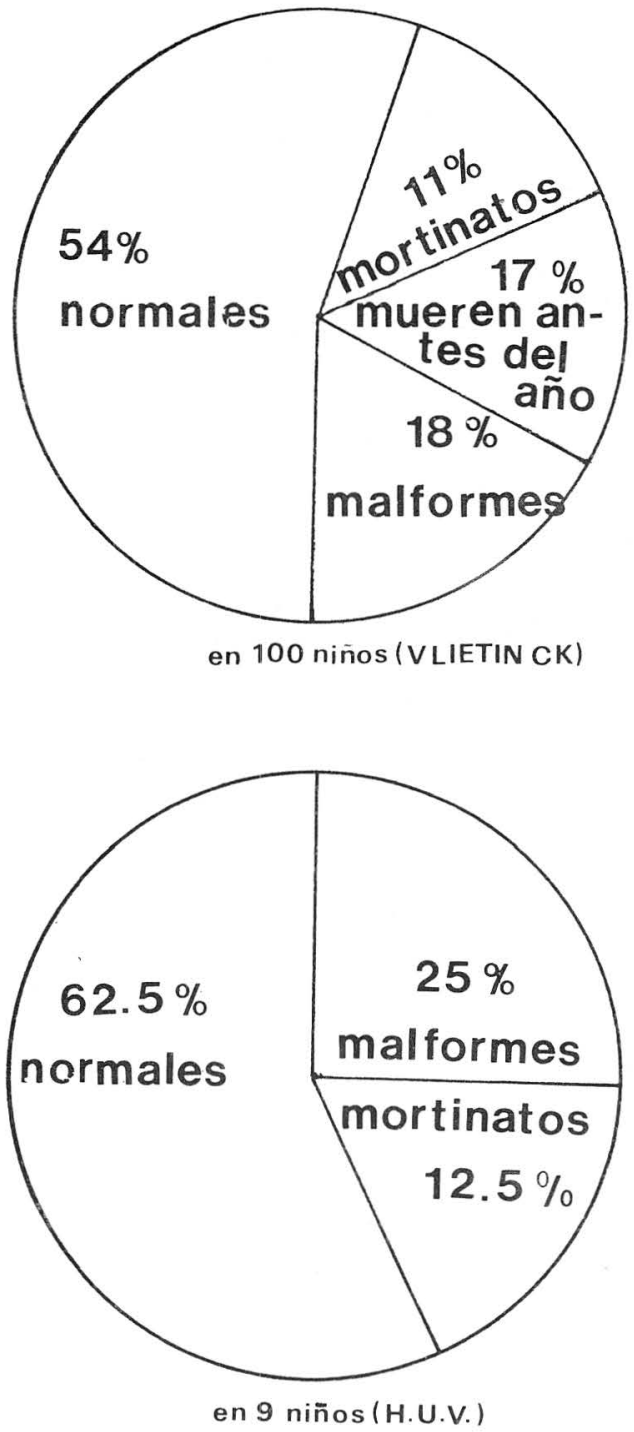

la mayoría de los autores. Mas aun, si comparamos graficamente nuestros resultados con los observados por Vlietinck (14), como puede verse en la figura No. 1, ellos se acercåran bastante a los obtenidos por este autor en una serie de 100 casos, y segun los cuales el niño con A.U.U. reduce a la mitad sus posibilidades de sobrevivir y ser normal. Basandose en observaciones si- 
milares Johnson (15) considera al niño con A.U.U. como de alto riesgo por lo cual debe ser tratado como tal desde el momento del nacimiento.

Estudios de segụimiento, como el realizado por Froehlich y Fujikura (16) sugieren que la A.U.U. es mas bien una malformación asociada a otras malformaciones, antes que su causa directa.

Nuestros resultados parciales muestran que tambien en nuestro medio existe tal asociación y que la A.U.U. debe ser establecida como un indicador para la busqueda de otras malformaciones. La presencia de alteraciones en los valores de inmunoglobulina, por ejemplo, deben seguirse investigando en nuestro medio, para tratar de establecer la posible relación de la A.U.U. con infección intrauterina, lo mismo que las posibles alteraciones cromosomicas y dermatoglificas en nuestros niños nacidos con ella (17).

En cuanto a la posible etiología de la A.U.U. no existe información definida; se acepta en general, que ello se debe a aplasia o atrofia del vaso faltante. (18) Un tercer mecanismo podría ser, segun Monie (19) la persistencia de la arteria umbilical única, normalmente transitoria, del desarrollo embrionario, en asociación con degeneración de la porción principal ya sea de la arteria umbilical derecha o de la izquierda.

\section{RESUMEN :}

Se presentan nueve casos de arteria umbilical única (A.U.U.), investigados entre 3.136 recien nacido.s Tal hallazgo coexiste en dos casos con malformaciones congénitas importantes demostradas de acuerdo con lo observado con anterioridad por otros autores. Por lo anterior se aconseja encacillar tales casos como casos de alto riesgo y proceder en consecuencia.

La presencia de alteraciones halladas en los valores de inrnunoglobulina y en el estudio cromosómico reafirma el concepto de que en tales casos influye un factor de alteración embrionaria.

\section{SUMARY:}

Nine cases of single umbilical artery was presented. This cases selected between. 3.126 new borns, presents two cases of true congenited malformations.

Fot that reaons, those cases must be considered as hight risk cases, and be agliged to proceed in consequence. Low values of inmunoglobulin and chromosomal fin-dings, thinks in embryonic cause.

\section{REFERENCIAS BIBLIOGRAFICAS}

1.- Vesalio, Citado por Faierman, E. Arch. Dis. Childhood 35: 285, 1960.

2.- Bauhin, C., (1620) Citado por Bridges, J. B. y Morton W.R.M., "The anatomical Record, 148: 103, 1964.

3.- Baudelocque, J. L'art des accouchements, 1: 250, 1822, Mecquicnon, Paris.

4.- Otto, A.W., (1830) Citado por Bridges, J. B. y Morton W.R.M., "The anatomical Record, 148: 103, 1964.

5.- Hyrtl, J. (1870) Citado por Bridges, J.B. y Morton W. R. M., "The anatomical Record, 148: 103, 1964.

6.- Benirschke, K., y Brown, W.H. Obst. \& Gynec., 6: 399, 1955.

7.- Papadatos, C., y Paschos, A., "Single Umbilical Artery and Congenital malformations"' Obst. \& Gynec., 26: 367, 1965.

8.- Seki, M; Strauss, L. "Absence of one um bilical artery: analysis of 60 cases with emphasis on associated developmental aberrations"' Arch. Pathol. 78: 446, 1964.

9.- Muller. G., et al. "L'artere ombilicale unique - A propos de 54 cas'" Bull. Soc. R. Belge Gy necol. Obst. 39: 333, 1969.

10.- Saroj, S., Srivstava, J. R. "Single umbilicale artery: its incidence and associated congenital malformations". Indian Pediatrics 5: 202, 1968.

11.- Giraud, J. R., et al. "Artere ombilicale unique et pathologie perinatale" Gyn. Obst. Paris, 70: 433, 1971.

12.- Seitzman, L. "The single umbilical artery", J. Newark Beth Israel Hosp. 15: 21, 1964. 
13.- Segovia, J. "Anomalias Vasculares del Cordón Umbilical" Rev. Obst. Gin. Venezuela 27: 421, 1967.

14.-- Vlietick, R.F. et al. "Signifance of the single umbilical artery: a clinical radiological chromosomal and dermatoglyphic study" Arch. Dis. Chil. 47: 639, 1972.

15.- Johnson, C., "The single umbilical artery and what it means"' Clinical Pediatrics 12: $367,1973$.

16.-- Froehlich, L., Fujikura, T. Follow-up of infants with single umbilical artery" Pediatrics 52: 6, 1973 .

17.- Khudr, G.; Benirschke, K. "Pure gonadal dysgenesis associated with a single umbilical artery - A case report" Obst. Gynec 38: 697,1971 .

18.- Bruniquel, M.G. "Preuves histologiques de l'erigine atresique habituelle dans le syndrome de l'artere ombilicale unique" Bull. Fed. Gynec. Obstet. Franç 23: 652, 1973.

19.- Monie, I, "Genesis of single umbilical artery" A.J. Obst. Gy nec. 108: 400, 1970. 